\title{
Fish fauna of Floresta Nacional de Ipanema, São Paulo State, Brazil
}

\author{
Welber Senteio Smith ${ }^{1,2,3}$, Renata Cassemiro Biagioni ${ }^{1}$ \& Letícia Halcsik $^{1}$ \\ ${ }^{1}$ Laboratório de Ecologia Estrutural e Funcional, Universidade Paulista - UNIP, Av. Independência, 210, \\ Jardim Éden, CEP 18087-101, Sorocaba, SP, Brazil \\ ${ }^{2}$ Departamento de Engenharia Ambienal, Universidade de Sorocaba - UNISO, \\ Rua Raposo Tavares, 92, Vila Assis, CEP 18023-000, Sorocaba, SP, Brazil \\ ${ }^{3}$ Corresponding author: Welber Senteio Smith,e-mail: welber_smith@uol.com.br
}

SMITH, W.S., BIAGIONI, R.C. \& HALCSIK, L. Fish fauna of Floresta Nacional de Ipanema, São Paulo State, Brazil. Biota Neotrop. 13(2): http://www.biotaneotropica.org.br/v13n2/en/abstract?inventory+bn01713022013

Abstract: The Floresta Nacional de Ipanema is located on the southeast of the state of São Paulo, Brazil, and belongs to the cities of Araçoiaba da Serra, Iperó and Capela do Alto. It extends over an area of 5,069 ha surrounded by several streams, small lakes, the Ipanema river and the Hedberg dam. The fish species in this area were sampled between 1993 and 2012 in several isolated studies, with the use of gill nets and sieves. Fifty species of fish were registered, 43 native and 7 non-native (Cyprinus carpio, Piaractus mesopotamicus, Brycon cephalus, Triportheus nematurus, Clarias gariepinus, Tilapia rendalli and Poecilia vivipara), distributed in seven Orders, 21 Families and 41 Genera. The present study aimed at cataloguing the fish species in the Ipanema National Forest. Handling and preservation actions will be based on the information obtained.

Keywords: Floresta Nacional de Ipanema, ichthyofauna, inventory, fish, conservation unit.

SMITH, W.S., BIAGIONI, R.C. \& HALCSIK, L. A ictiofauna da Floresta Nacional de Ipanema, Estado de São Paulo, Brasil. Biota Neotrop. 13(2): http://www.biotaneotropica.org.br/v13n2/pt/abstract?inventory+ bn01713022013

Resumo: A Floresta Nacional de Ipanema está localizada no sudoeste do estado de São Paulo, Brasil e pertence às cidades de Araçoiaba da Serra, Iperó e Capela do Alto. Estende-se por uma área de 5, 069 ha, onde ocorrem diversos riachos, pequenos lagos, o rio Ipanema e a represa de Hedberg. As espécies de peixes dessa unidade de conservação foram amostradas entre 1993 e 2012 em vários estudos realizados, com o uso de redes de espera e peneiras. Foram registradas 50 espécies de peixes, 43 nativas e 7 não-nativas (Cyprinus carpio, Piaractus mesopotamicus, Brycon cephalus, Triportheus nematurus, Clarias gariepinus, Tilapia rendalli e Poecilia vivipara), distribuídas em sete ordens, 21 famílias e 41 gêneros. O estudo teve como objetivo catalogar as espécies de peixes da Floresta Nacional de Ipanema. Ações de manejo e preservação deverão ser baseadas nas informações obtidas. Palavras-chave: Floresta Nacional de Ipanema, ictiofauna, inventário, peixe, unidade de conservação. 


\section{Introduction}

Currently, there are 391 species described for São Paulo state, representing about $15 \%$ of freshwater fish species of the Brazil (Oyakawa \& Menezes 2011). Recently Smith et al. 2007 registered 71 species of fishes in the Sorocaba river basin, where it is located the Floresta Nacional de Ipanema.

The Floresta Nacional de Ipanema (FLONA de Ipanema) is a national conservation unit created in 1992, which is maintained by the Instituto Nacional do Meio Ambiente e Recursos Naturais Renováveis (IBAMA). It extends over an area of 5,069 ha, with 1,388 ha being covered with vegetation (Smith \& Regalado 2008).

The three main watercourses that run across the area are the Ipanema, Verde and Ribeirão do Ferro streams. Ipanema and Verde streams make up the basin of Ipanema River, and Ribeirão do Ferro and other streams located within the conservation unit make up the microbasin of Ribeirão do Ferro. Besides these watercourses, there is the hydrographic basin of the Ipero River, located in the western portion of the Araçoiaba Mount.

Significant environmental impacts were identified in the microbasins comprised in the present study. The replacement of natural vegetation with pasture fields is noticeable in the Iperó River, which causes erosion and silting of watercourses. This is also found in the Ipanema River, where effluent discharge and use of pesticides are frequent. The microbasin of Ribeirão do Ferro, in turn, is in better conditions since it is located in the Araçoiaba Mount. However, this small stream is also affected by adverse conditions, such as the many dams in its bed and the silting resulting from a landslide of waste on its left edge next to the area of limestone mining, and the suppression of riparian vegetation in the lowest areas of the Araçoiaba Mount (downstream).

The above finding deserves attention, because the little-known fish species of the region may be endangered. The ichthyofauna of the basins located in the Floresta Nacional de Ipanema was first registred by Georg Heinrich von Langsdorff, who visited the region, in 1825, and identified the following fish species: "jundiá" (Rhamdia quelen), "mandiuva" (Pimelodus maculatus), "traíra" (Hoplias malabaricus) and "tuvira" (Gymnotus carapo) (Expedição... 1821-1829).

The knowledge of the fish fauna of this region is mainly due to the work of Bernardo \& Barrella (1999), Smith (1999), Smith \& Marciano (2000) well as studies for the preparation of the Management Plan in 2003 (Brasil 2003). More recently, the studies of Smith (2003), Smith et al. (2007), Smith \& Regalado (2008), and Smith et al. (2009) were conducted. Being conservation areas considered place of survival within a larger unit changed (Sarmento-Soares \& Martins-Pinheiro, 2007), knowledge of existing species is critical. So, the present study aimed at cataloguing the fish species in the conservation unit based on the above mentioned studies and on the sampling of species to provide information as a basis for handling and preservation actions.

\section{Material and Methods}

The Floresta Nacional de Ipanema is located between latitudes $23^{\circ} 25^{\prime}$ and $23^{\circ} 28^{\prime} \mathrm{S}$ and longitudes $47^{\circ} 33^{\prime}$ and $47^{\circ} 40^{\prime} \mathrm{W}$ in an area of 5,180 ha belonging the municipalities of Iperó, Araçoiaba da Serra and Capela do Alto, on the southeast of the state of São Paulo, $125 \mathrm{~km}$ far from the capital, São Paulo.

Various streams emerge from the Araçoiaba Mount (Figure 1), and the largest one is called Ribeirão do Ferro (Salazar 1998). This stream is formed by three small irregular streams of water, in which confluence is the "Cobra" Lagoon, a reservoir of Ribeirão do Ferro, constructed in the beginning of 1970. There are other lagoons besides the Cobra Lagoon. The Ipanema River, a major tributary of the Sorocaba River, flows east of the Araçoiaba Mount, forming the Hedberg Dam, constructed in 1818 (Salazar 1998). All these waterways make up the hydrographic basin of the Sorocaba River, main tributary of the left bank of the Tietê River.

Fish were collected in some stretches of Ribeirão do Ferro and Verde rivers, in one stretch of the Ipanema River (dam and downstream the dam), in the Hedberg Dam, in the Limão and Cobra lagoons and in other lagoons that occur in the FLONA (Table 1). The fish were captured with sieves and nets with meshes of 3, 4, 6, 7, 8,10 and $12 \mathrm{~cm}$ between opposing knots. The organisms collected were stored in the fish collection of the biology museum of the Universidade Paulista - Campus Sorocaba.

\section{Results}

A total of 50 species distributed in seven orders, 21 families and 41 genera were identified. The taxonomic list of identified species is shown in Table 2. Most of these species belonged to the Characiformes order (25 species) and the Siluriformes (18 species). The most represented families are: Characidae, Loricariidae, Heptapteridae and Callichthyidae with 15, 6, 3 and 3 species, respectively. Of the total identified species, 43 are native and seven are considered non-native. Regarding the distribution of the fish fauna, 27 species are widely distributed throughout the conservation area while 23 were recorded to a particular habitat (Table 3). Besides, non-native species present in the area are concentrated in lentic environment as the Hedberg reservoir and Cobra lagoon, except Tilapia rendalli which is widely

Table 1. Sampling stations sampled in the Floresta Nacional de Ipanema with their ecological status and geographic location.

\begin{tabular}{|c|c|c|}
\hline $\begin{array}{c}\text { Sampling } \\
\text { stations }\end{array}$ & $\begin{array}{c}\text { Ecological } \\
\text { status }\end{array}$ & $\begin{array}{c}\text { Geographical } \\
\text { coordinates }\end{array}$ \\
\hline Ipa01 - Ipanema River & $\begin{array}{c}\text { class } 2 \text { river, lotic, } \\
\text { high current }\end{array}$ & $\begin{array}{c}\text { 23K } 239172 \\
\text { UTM } 7399430\end{array}$ \\
\hline Ipa02 - Ipanema River & $\begin{array}{c}\text { class } 2 \text { river, lotic, } \\
\text { high current }\end{array}$ & $\begin{array}{l}\text { 23K } 0234693 \\
\text { UTM } 7407007\end{array}$ \\
\hline Ipa03 - Verde River & $\begin{array}{c}\text { stream, lotic, low } \\
\text { current }\end{array}$ & $\begin{array}{c}\text { 23K } 236768 \\
\text { UTM } 7404492\end{array}$ \\
\hline Ipa04 - Verde River & $\begin{array}{l}\text { stream, lotic, low } \\
\text { current }\end{array}$ & $\begin{array}{c}\text { 23K } 235202 \\
\text { UTM } 7403739\end{array}$ \\
\hline Ipa05 - Hedberg Dam & reservoir, lentic & $\begin{array}{c}\text { 23K } 235171 \\
\text { UTM } 7406740\end{array}$ \\
\hline Ipa06 - Hedberg Dam & reservoir, lentic & $\begin{array}{c}\text { 23K } 0234518 \\
\text { UTM } 7406543\end{array}$ \\
\hline Ipa07 - Hedberg Dam & reservoir, lentic & $\begin{array}{l}\text { 23K } 0234859 \\
\text { UTM } 7406967\end{array}$ \\
\hline $\begin{array}{l}\text { Ipa08 - Ribeirão do } \\
\text { Ferro Stream }\end{array}$ & $\begin{array}{c}\text { stream, lotic, } \\
\text { moderate current }\end{array}$ & $\begin{array}{l}\text { 23K } 0232070 \\
\text { UTM } 7406301\end{array}$ \\
\hline $\begin{array}{l}\text { Ipa } 09 \text { - Ribeirão do } \\
\text { Ferro Stream }\end{array}$ & $\begin{array}{c}\text { stream, lotic, } \\
\text { moderate current }\end{array}$ & $\begin{array}{c}\text { 23K } 0231146 \\
\text { UTM } 7405348\end{array}$ \\
\hline Ipa10 - Cobra Lagoon & lentic environment & $\begin{array}{c}\text { 23K } 2330394 \\
\text { UTM } 7404885\end{array}$ \\
\hline Ipa11 - Limão Lagoon & lentic environment & $\begin{array}{c}\text { 23K } 233765 \\
\text { UTM } 7407117\end{array}$ \\
\hline Ipa 12 - Lagoon & lentic environment & $\begin{array}{c}\text { 23k } 0234479 \\
\text { UTM } 7406472\end{array}$ \\
\hline Ipa 13 - Lagoon & lentic environment & $\begin{array}{l}\text { 23k } 0234284 \\
\text { UTM } 7406554\end{array}$ \\
\hline Ipa 14 - Lagoon & lentic environment & $\begin{array}{c}\text { 23k0234681 } \\
\text { UTM } 7404199\end{array}$ \\
\hline
\end{tabular}




\section{FLONA DE IPANEMA}

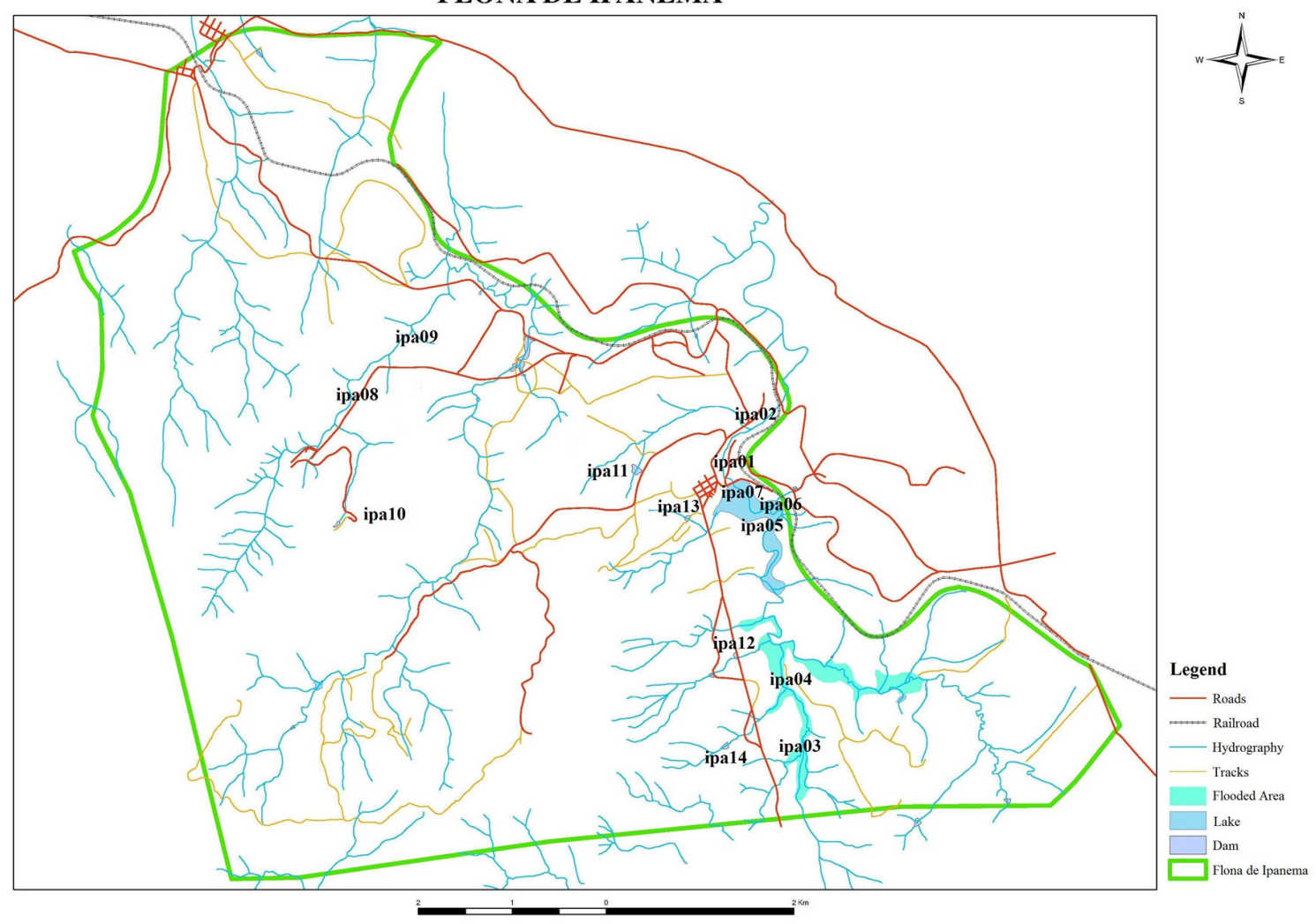

Figure 1. Hidrographic system of the Floresta Nacional de Ipanema.

Table 2. Taxonomic list of fish species from the Floresta Nacional de Ipanema.

\begin{tabular}{|c|c|}
\hline & Species \\
\hline \multicolumn{2}{|l|}{ Characiformes } \\
\hline \multirow[t]{15}{*}{ Characidae } & Astyanax fasciatus (Cuvier, 1819) \\
\hline & Astyanax altiparanae Garutti and Britski, 2000 \\
\hline & Astyanax eigenmanniorum (Cope, 1894) \\
\hline & Astyanax paranae Eigenmann, 1914 \\
\hline & Astyanax scabripinnis (Jenyns, 1842) \\
\hline & Hyphessobrycon anisitsi (Eigenmann, 1907) \\
\hline & Hemigrammus marginatus Ellis, 1911 \\
\hline & Serrapinnus notomelas (Eigenmann, 1915) \\
\hline & Odontostilbe microcephala Eigenmann, 1907 \\
\hline & Bryconamericus stramineus Eigenmann, 1908 \\
\hline & Brycon cephalus* (Günther, 1869) \\
\hline & Oligosarcus pintoi Campos, 1945 \\
\hline & Acestrorhynchus lacustris (Lütken, 1875) \\
\hline & Salminus hilarii Valenciennes, 1850 \\
\hline & Triportheus nematurus * (Kner, 1858) \\
\hline \multicolumn{2}{|l|}{ Crenuchidae } \\
\hline & Characidium zebra Eigenmann, 1909 \\
\hline \multirow[t]{2}{*}{ Serrasalmidae } & \\
\hline & Piaractus mesopotamicus * (Holmberg, 1887) \\
\hline \multirow[t]{2}{*}{ Erythrinidae } & \\
\hline & Hoplias malabaricus (Block, 1794) \\
\hline \multirow[t]{2}{*}{ Prochilodontidae } & Prochilodus lineatus (Valenciennes,1836) \\
\hline & Prochilodus vimboides Kner, 1859 \\
\hline Curimatidae & Steindachnerina insculpta (Fernández-Yépez, 1948) \\
\hline
\end{tabular}

\footnotetext{
* non-native specie
} 
Table 2. Continued...

\begin{tabular}{|c|c|}
\hline & Species \\
\hline & Cyphocharax modestus (Fernández-Yépez, 1948) \\
\hline Anostomidae & Leporinus obtusidens (Valenciennes, 1836) \\
\hline \multicolumn{2}{|l|}{ Parodontidae } \\
\hline & Parodon nasus Kner, 1859 \\
\hline & Apareiodon affinis (Steindachner, 1879) \\
\hline \multicolumn{2}{|c|}{ CYPRINIFORMES } \\
\hline Cyprinidae & Cyprinus carpio* Linnaeus, 1758 \\
\hline \multicolumn{2}{|l|}{ SILURIFORMES } \\
\hline \multicolumn{2}{|l|}{ Callichthyidae } \\
\hline & Corydoras aeneus (Gill, 1858) \\
\hline & Corydoras flaveolus Ihering, 1911 \\
\hline & Hoplosternum littorale (Hancock, 1828) \\
\hline \multicolumn{2}{|l|}{ Cetopsidae } \\
\hline & Cetopsis gobioides Kner, 1857 \\
\hline \multicolumn{2}{|l|}{ Clariidae } \\
\hline & Clarias gariepinus* (Burchell, 1822) \\
\hline \multicolumn{2}{|l|}{ Pimelodidae } \\
\hline & Pimelodus maculatus La Cèpede, 1803 \\
\hline & Iheringichthys syi Azpelicueta \& Britski, 2012 \\
\hline \multicolumn{2}{|l|}{ Heptapteridae } \\
\hline & Imparfinis mirini Haseman, 1911 \\
\hline & Pimelodella rudolphi (Miranda-Ribeiro, 1918) \\
\hline & Rhamdia quelen (Quoy and Gaimard, 1824) \\
\hline \multicolumn{2}{|c|}{ Pseudopimelodidae } \\
\hline & Microglanis garavelloi Shibatta and Benine, 2005 \\
\hline \multicolumn{2}{|c|}{ Trichomycteridae } \\
\hline \multirow[t]{7}{*}{ Loricariidae } & Trichomycterus iheringi \\
\hline & Hisonotus depressicauda (Miranda-Ribeiro, 1918) \\
\hline & Hypostomus ancistroides (Ihering, 1911) \\
\hline & Hypostomus margaritifer (Regan, 1908) \\
\hline & Hypostomus sp. \\
\hline & Rineloricaria latirostris (Boulenger, 1900) \\
\hline & Rineloricaria sp. \\
\hline \multicolumn{2}{|c|}{ GYMNOTIFORMES } \\
\hline Gymnotidae & Gymnotus carapo Linnaeus, 1758 \\
\hline \multicolumn{2}{|l|}{ PERCIFORMES } \\
\hline \multicolumn{2}{|l|}{ Cichlidae } \\
\hline & Geophagus brasiliensis (Quoy and Gaimard, 1824) \\
\hline & Tilapia rendalli* (Boulenger, 1897) \\
\hline \multicolumn{2}{|c|}{ Cyprinodontiformes } \\
\hline \multirow[t]{2}{*}{ Poeciliidae } & Phalloceros reisi Lucinda, 2008 \\
\hline & Poecilia vivipara Block and Schneider, 1801 \\
\hline \multicolumn{2}{|c|}{ SYNBRANCHIFORMES } \\
\hline Synbranchidae & Synbranchus marmoratus Bloch, 1795 \\
\hline
\end{tabular}

* non-native specie

distributed by the numerous water bodies of the area. According to Table 3 is possible to see eight species that exhibit migratory habit, eight exhibit parental care and two, both Poeciliidae, have internal fertilization. Regarding the feeding habits of the species found in the Floresta Nacional de Ipanema, predominated omnivorous and insectivorous species (14 and 13 species respectively). One of the identified species (Prochilodus vimboides) is considered vulnerable in the state according to the book of threatened fauna in the State of São Paulo (Bressan et al. 2009). Other features and details of the distribution of species are included in Table 3.

\section{Discussion}

The ichthyofauna composition is the one expected for non estuarine waters of the neotropical region (Lowe-McConell 1987, Castro \& Menezes 1998). Among the various water ecosystems in this conservation unit, we can cite the streams with moderate to high flows, waterfall stretches, gravelly soil, in some cases consolidated substratum, great concentration of allochthonous and autochthonous insects and the typical herbaceous and shrubby vegetation, which 
Fish fauna of Flona de Ipanema

Table 3. Fish species occurring in the Floresta Nacional de Ipanema, with their characteristics and distribution.

\begin{tabular}{|c|c|c|c|c|c|}
\hline Species & $\begin{array}{l}\text { Vulgar } \\
\text { names }\end{array}$ & Feeding habit & Preferred habitats & Reproduction & Distribution \\
\hline Astyanax altiparanae & $\begin{array}{l}\text { lambari do } \\
\text { rabo amarelo }\end{array}$ & omnivorous & $\begin{array}{l}\text { backwaters, near the } \\
\text { margin }\end{array}$ & $\begin{array}{l}\text { external fertilization, without } \\
\text { parental care, not migratory }\end{array}$ & wide \\
\hline Astyanax fasciatus & $\begin{array}{l}\text { lambari do } \\
\text { rabo vermelho }\end{array}$ & omnivorous & vegetation on the margins & $\begin{array}{l}\text { external fertilization, without } \\
\text { parental care }\end{array}$ & wide \\
\hline Astyanax scabripinnis & lambari & insectivorous & backwaters of streams & $\begin{array}{l}\text { external fertilization, without } \\
\text { parental care, not migratory }\end{array}$ & restricted \\
\hline Astyanax eigenmanniorum & lambari & insectivorous & benthopelagic & $\begin{array}{l}\text { external fertilization, without } \\
\text { parental care, not migratory }\end{array}$ & restricted \\
\hline Astyanax paranae & lambari & insectivorous & benthopelagic & $\begin{array}{l}\text { external fertilization, without } \\
\text { parental care, not migratory }\end{array}$ & restricted \\
\hline Hemigrammus marginatus & piaba & omnivorouss & vegetation on the margins & $\begin{array}{l}\text { external fertilization, without } \\
\text { parental care, not migratory }\end{array}$ & restricted \\
\hline Hyphessobrycon anisitsi & tetra & $\begin{array}{l}\text { insectivorous } \\
\text { herbivore }\end{array}$ & benthopelagic & $\begin{array}{l}\text { external fertilization, without } \\
\text { parental care }\end{array}$ & restricted \\
\hline Serrapinnus notomelas & piaba & omnivorous & vegetation on the margins & $\begin{array}{l}\text { external fertilization, without } \\
\text { parental care, not migratory }\end{array}$ & wide \\
\hline Odontostilbe microcephala & pequira & omnivorous & benthopelagic & $\begin{array}{l}\text { external fertilization, without } \\
\text { parental care, not migratory }\end{array}$ & restricted \\
\hline Bryconamericus stramineus & pequira & omnivorous & $\begin{array}{l}\text { backwaters or currents } \\
\text { between the grass, } \\
\text { benthopelagic }\end{array}$ & $\begin{array}{l}\text { external fertilization, without } \\
\text { parental care, not migratory }\end{array}$ & restricted \\
\hline Brycon cephalus ${ }^{1}$ & matrinxã & omnivorous & benthopelagic & $\begin{array}{l}\text { external fertilization, with } \\
\text { parental care migratory }\end{array}$ & wide \\
\hline Oligosarcus pintoi & $\begin{array}{l}\text { peixe- } \\
\text { cachorro }\end{array}$ & carnivorous & $\begin{array}{l}\text { roots of the marginal } \\
\text { vegetation }\end{array}$ & $\begin{array}{l}\text { external fertilization, without } \\
\text { parental care, not migratory }\end{array}$ & wide \\
\hline Characidium zebra & canivete & insectivorous & $\begin{array}{l}\text { rocky substrate, } \\
\text { benthopelagic, }\end{array}$ & $\begin{array}{l}\text { external fertilization, without } \\
\text { parental care, not migratory }\end{array}$ & restricted \\
\hline Acestrorhynchus lacustris & cadela & piscivore & backwaters & $\begin{array}{l}\text { external fertilization, without } \\
\text { parental care, not migratory }\end{array}$ & wide \\
\hline Salminus hilarii & tabarana & carnivorous & currents & $\begin{array}{l}\text { external fertilization, migra- } \\
\text { tory, without parental care }\end{array}$ & wide \\
\hline Triportheus nematurus ${ }^{1}$ & sardinha & insectivorous & currents & $\begin{array}{l}\text { external fertilization, migra- } \\
\text { tory, without parental care }\end{array}$ & restricted \\
\hline Piaractus mesopotamicus $^{\mathbf{1}}$ & pacu & herbivore & demersal & $\begin{array}{l}\text { external fertilization, migra- } \\
\text { tory, without parental care }\end{array}$ & restricted \\
\hline Steindachnerina insculptaSaguirú & saguirú & iliophagous & backwaters, background & $\begin{array}{l}\text { external fertilization, without } \\
\text { parental care, not migratory }\end{array}$ & wide \\
\hline Cyphocharax modestus & saguirú & iliophagous & backwaters, background & $\begin{array}{l}\text { external fertilization, without } \\
\text { parental care, not migratory }\end{array}$ & wide \\
\hline Prochilodus lineatus & curimbatá & iliophagous & current & $\begin{array}{l}\text { external fertilization, migra- } \\
\text { tory, without parental care }\end{array}$ & restricted \\
\hline Prochilodus vimboides $^{2}$ & curimbatá & iliophagous & current & $\begin{array}{l}\text { external fertilization, migra- } \\
\text { tory, without parental care }\end{array}$ & restricted \\
\hline Hoplias malabaricus & traíra & carnivorous & backwaters, lagoons & $\begin{array}{l}\text { external fertilization, with } \\
\text { parental care, not migratory }\end{array}$ & wide \\
\hline Leporinus obtusidens & piava & herbivore & lotic environments & $\begin{array}{l}\text { external fertilization, without } \\
\text { parental care, migratory }\end{array}$ & wide \\
\hline Parodon nasus & canivete & iliophagous & $\begin{array}{l}\text { marginal areas, current, } \\
\text { gritty background }\end{array}$ & $\begin{array}{l}\text { external fertilization, without } \\
\text { parental care, not migratory }\end{array}$ & wide \\
\hline Apareiodon affinis & canivete & detritivore & $\begin{array}{l}\text { benthopelagic, lotic and } \\
\text { lentic environment }\end{array}$ & $\begin{array}{l}\text { external fertilization, without } \\
\text { parental care, not migratory }\end{array}$ & restricted \\
\hline 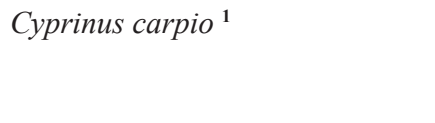 & carpa & $\begin{array}{l}\text { herbivore- } \\
\text { detritivore and } \\
\text { zoobenthos }\end{array}$ & benthopelagic & $\begin{array}{l}\text { external fertilization, without } \\
\text { parental care, not migratory }\end{array}$ & restricted \\
\hline Corydoras aeneus & ronquinha & omnivorous & $\begin{array}{l}\text { clear water, backwaters or } \\
\text { currents }\end{array}$ & $\begin{array}{l}\text { external fertilization, without } \\
\text { parental care, not migratory }\end{array}$ & wide \\
\hline
\end{tabular}

${ }^{1}$ Introduced species / non-native. ${ }^{2}$ Specie in vulnerable state according to Bressan et al. ( 2009). 
Table 3. Continued...

\begin{tabular}{|c|c|c|c|c|c|}
\hline Species & $\begin{array}{l}\text { Vulgar } \\
\text { names }\end{array}$ & Feeding habit & Preferred habitats & Reproduction & Distribution \\
\hline Corydoras flaveolus & ronquinha & omnivorous & $\begin{array}{l}\text { clear water, backwaters or } \\
\text { currents }\end{array}$ & $\begin{array}{l}\text { external fertilization, without } \\
\text { parental care, not migratory }\end{array}$ & restricted \\
\hline Cetopsis gobioides & candiru & insectivorous & current and demersal & $\begin{array}{l}\text { external fertilization, without } \\
\text { parental care, not migratory }\end{array}$ & restricted \\
\hline Clarias gariepinus ${ }^{1}$ & bagre africano & omnivorous & benthopelagic & $\begin{array}{l}\text { external fertilization, } \\
\text { without parental care, with } \\
\text { lateral migration }\end{array}$ & restricted \\
\hline Hypostomus ancistroides & cascudo & $\begin{array}{l}\text { herbivore- } \\
\text { detritivore }\end{array}$ & river rapids, background & $\begin{array}{l}\text { external fertilization, with } \\
\text { parental care, not migratory }\end{array}$ & wide \\
\hline Hypostomus margaritifer & cascudo & $\begin{array}{l}\text { herbivore- } \\
\text { detritivore }\end{array}$ & river rapids, background & $\begin{array}{l}\text { external fertilization, without } \\
\text { parental care, not migratory }\end{array}$ & wide \\
\hline Hypostomus sp. & cascudo & $\begin{array}{l}\text { herbivore- } \\
\text { detritivore }\end{array}$ & river rapids, background & $\begin{array}{l}\text { external fertilization, without } \\
\text { parental care, not migratory }\end{array}$ & wide \\
\hline Rineloricaria latirostris & cascudo & detritivore & current, rocks and trunk & $\begin{array}{l}\text { external fertilization, without } \\
\text { parental care, not migratory }\end{array}$ & restricted \\
\hline Rineloricaria sp. & cascudo & detritivore & current, rocks and trunk & $\begin{array}{l}\text { external fertilization, without } \\
\text { parental care, not migratory }\end{array}$ & restricted \\
\hline Hisonotus depressicauda & limpa-vidro & detritivore & vegetation on the margins & $\begin{array}{l}\text { external fertilization, without } \\
\text { parental care, not migratory }\end{array}$ & restricted \\
\hline Hoplosternum litoralle & caborja & iliophagous & backwaters, background & $\begin{array}{l}\text { external fertilization with } \\
\text { parental care }\end{array}$ & wide \\
\hline Imparfinis mirini & bagrinho & insectivorous & $\begin{array}{l}\text { current, pebble, gravel and } \\
\text { grit background }\end{array}$ & $\begin{array}{l}\text { not migratory, external fertili- } \\
\text { zation with parental care }\end{array}$ & wide \\
\hline Microglanis garavelloi & bagrinho & insectivorous & vegetation on the margins & $\begin{array}{l}\text { not migratory } \\
\text { external fertilization }\end{array}$ & restricted \\
\hline Pimelodella rudolphi & mandizinho & insectivorous & $\begin{array}{l}\text { Current, pebble } \\
\text { background and demersal }\end{array}$ & $\begin{array}{l}\text { external fertilization, without } \\
\text { parental care, not migratory }\end{array}$ & restricted \\
\hline Pimelodus maculatus & $\begin{array}{l}\text { mandi- } \\
\text { pintado }\end{array}$ & insectivorous & current, background & $\begin{array}{l}\text { external fertilization, without } \\
\text { parental care, not migratory }\end{array}$ & wide \\
\hline Iheringichthys syi & mandi & $\begin{array}{l}\text { carnivorous, } \\
\text { insectivorous }\end{array}$ & rivers, background & $\begin{array}{l}\text { external fertilization, without } \\
\text { parental care, not migratory }\end{array}$ & wide \\
\hline Rhamdia quelen & bagre & omnivorous & low current, background & $\begin{array}{l}\text { external fertilization, with } \\
\text { parental care, not migratory }\end{array}$ & wide \\
\hline Trichomycterus sp. & sobe-serra & insectivorous & $\begin{array}{l}\text { current, grit and gravel } \\
\text { background }\end{array}$ & $\begin{array}{l}\text { external fertilization, without } \\
\text { parental care, not migratory }\end{array}$ & restricted \\
\hline Gymnotus carapo & tuvira & carnivorous & vegetation on the margins & $\begin{array}{l}\text { external fertilization, without } \\
\text { parental care, not migratory }\end{array}$ & wide \\
\hline Geophagus brasiliensis & cará & omnivorous & margins & $\begin{array}{l}\text { external fertilization, with } \\
\text { parental care, not migratory }\end{array}$ & wide \\
\hline Tilapia rendalli ${ }^{\mathbf{1}}$ & tilápia & herbivore & margins & $\begin{array}{l}\text { external fertilization, with } \\
\text { parental care, not migratory }\end{array}$ & wide \\
\hline Phaloceros reisi Barrigudinho, & barrigudinho & omnivorous & margins, shallow water & $\begin{array}{l}\text { internal fertilization, not } \\
\text { migratory }\end{array}$ & wide \\
\hline Poecilia vivipara ${ }^{1}$ & barrigudinho & omnivorous & margins, shallow water & $\begin{array}{l}\text { internal fertilization, not } \\
\text { migratory }\end{array}$ & wide \\
\hline Synbranchus marmoratus & mussum & carnivorous & $\begin{array}{l}\text { lentic environment, cleft in } \\
\text { the margins }\end{array}$ & $\begin{array}{l}\text { external fertilization, not } \\
\text { migratory }\end{array}$ & wide \\
\hline
\end{tabular}

${ }^{1}$ Introduced species / non-native. ${ }^{2}$ Specie in vulnerable state according to Bressan et al. ( 2009).

favors the presence of small characids ("lambaris"), loricariids ("cascudos"), and catfish.

The ichthyofauna of the region consists of small-sized fish, such as "carás", "lambaris", "cascudos" and small catfish, which depend on the riparian vegetation for their food source of allochthonous material. The presences of Astyanax scabripinnis, a species typically found in streams, was registered (Castro \& Casatti 1997). The streams located in the conservation unit are ecosystems with a little known ichthyofauna (Castro \& Menezes 1998, Menezes et al. 2007). According Oyakawa et al. (2006), this lack of knowledge is related to the fact that the fish are small and uninteresting streams for fishing, besides their cryptic habits and occur in aquatic environments quite restricted. The author also highlights the difficulty in sampling all species present in a given stretch of river. 
According to Bressan et al. (2009) the species Prochilodus vimboides and Bunocephalus larai occur in vulnerable state in the area of the Floresta Nacional de Ipanema. However, no individual from Bunocephalus larai was collected during this work.

The predominant species in these rivers, including Verde and Ipanema rivers, are the "cará" (Geophagus brasiliensis), the "lambaris" (Astyanax altiparanae and A. fasciatus), the "saguirú" (Cyphocharax modestus and Steindachnerina insculpta), the lacustris catfish (Rhamdia quelen, Pimelodus maculatus and Iheringichthys labrosus), the "cascudo" (Hypostomus ancistroides), the "peixecadela" (Acestrorhynchus lacustris) and the "guarú" (Phalloceros reisi).

In addition to rivers and streams there are several lagoons as the Cobra and the Limão lagoons with several fish species. Fish of five species were identified there, as follows: "cará" (Geophagus brasiliensis), "lambari" (Astyanax altiparanae), "tilapia" (Tilapia rendalli), "barrigudinho" (Phalloceros reisi) and "cascudo" (Hypostomus ancistroides). Because these lagoons are located in the headwater of rivers they have reduced diversity of species.

The Hedberg Dam, constructed in 1818, showed an ichthyofauna typical of lentic environment mainly formed by "saguirus" (Cyphocharax modestus and Steindachnerina insculpta), "carás" (Geophagus brasiliensis) and "lambaris" (Astyanax fasciatus and A. altiparanae). Moreover, species from typical lotic environments have been captured, such as the "curimbatá" (rheophilic species) and the "cascudo" (Hypostomus ancistroides), that are supposed to reproduce in the upstream stretches or in tributaries such as the Verde River. Being an old dam, the Hedberg Dam has a highly structured fish community. According to Lowe-McConnell (1987), it takes around 5 to 15 years for a fish community to reach stability.

This dam has been transformed into a pay to fish lake, and several species were added to this environment, such as the Tilapia rendalli, Piaractus mesopotamicus, Brycon cephalus and Cyprinus carpio (Smith 2003), which may contribute to the decreased stability of this fish community. Currently, although the fishing has been disabled, in addition to these species, it is also possible to verify the presence of the "catfish", Clarias gariepinus. This is because aquatic environments located in conservation areas, although legally protected, are subject to threats of a species is introduced elsewhere in the basin, because a water system tends to be virtually connected with all parts and subsystems, from the source to the mouth, the main channel of the lagoons (Agostinho et al. 2006).

\section{Acknowledgements}

We acknowledge the ICMBio for the logistic support, Dr. Heraldo Britski for confirming the identified species, Dr. Walter Barrella for the comments and Dr. Luciano Bonatti Regalado for the support and map.

\section{References}

AGOSTINHO, A.A., PELICICE, F.M. \& JÚliO JUNIOR, H.F. 2006. Biodiversidade e introdução de espécies de peixes: unidades de conservação. Unidades de conservação: ações para valorização da biodiversidade. Instituto Ambiental do Paraná, Curitiba, p.95-117.

BERNARDO, F.O. \& BARRELLA, W. 1999. Estudo comparativo da comunidade de peixes em duas lagoas existentes na Floresta Nacional de Ipanema, Iperó, SP. São Paulo. Relatório Final do Programa de Iniciação Científica PUC-SP/CNPq.
BRASIL. Ministério do Meio Ambiente - MMA. 2003. Plano de Manejo da Floresta Nacional de Ipanema. http://www.icmbio.gov.br/portal/images/ stories/imgs-unidades-coservacao/flona_ipanema.pdf (último acesso em 21/08/2012).

BRESSAN, P.M., KIERULFF, M.C.M. \& SUGIEDA, A.M. 2009. Fauna ameaçada de extinção no estado de São Paulo: Vertebrados. Governo do Estado de São Paulo, Fundação Parque Zoológico de São Paulo, Secretaria do Meio Ambiente, São Paulo, 2009.

CASTRO, R.M.C. \& CASATTI L. 1997. The fish fauna from a small forest stream of the upper Paraná River Basin, southeastern Brazil. Ichthyol. Explor. Freshwaters. 7(4):337-352.

CASTRO, R.M.C. \& MENEZES N.A. 1998. Estudo diagnóstico da diversidade de peixes do Estado de São Paulo. In Biodiversidade do Estado de São Paulo, Brasil: Síntese do conhecimento ao final do século XX, v.6 Vertebrados. (C.A. Joly \& C.E.M. Bicudo, orgs.). Winnergraph, FAPESP, São Paulo, p.1-13.

EXPEDIÇÃO Langsdorff ao Brasil. 1821-1829. Rio de Janeiro, Edições Alumbramento. v.1, Aquarelas e Desenhos de Rugendas, 154p., v.2, Aquarelas e Desenhos de Taunay, 159p., v.3, Aquarelas e Desenhos de Florence, 134p.

LOWE-McCONNELL, R.H.L. 1987. Ecological Studies in Tropical Fish Communities. Cambridge University Press, Cambridge, 382p. http:// dx.doi.org/10.1017/CBO9780511721892

MENEZES, N.A., WEITZMAN, S., OYAKAWA, O.T., LIMA, F., CASTRO, R. \& WEITZMAN, M. 2007. Peixes de água doce da mata atlântica. Museu de Zoologia, USP, Conservação Internacional, FAPESP, CNPq, São Paulo.

OYAKAWA, O.T., AKAMA, A., MAUTARI, K.C. \& NOLASCO, J.C. 2006. Peixes de riachos da Mata Atlântica nas Unidades de Conservação do Vale do Rio Ribeira de Iguape no estado de São Paulo. Editora Neotropica, São Paulo, 201p.

OYAKAWA, O.T. \& MENEZES, N.A. 2011. Checklist dos peixes de água doce do Estado de São Paulo, Brasil. Biota Neotrop. 11(1a): http://www. biotaneotropica.org.br/v11n1a/pt/abstract?inventory+bn0021101a2011 (último acesso em 21/08/2012).

SALAZAR, J.M. 1998. Araçoiaba and Ipanema: a história daquela maravilhosa região, desde as forjas de Afonso Sardinha até a Real Fábrica de Ferro. Digipel, Sorocaba, 120p.

SARMENTO-SOARES, L.M. \& MARTINS-PINHEIRO, R.F. 2007. A importância da ictiologia na definição de Unidades de Conservação. Bol. Soc. Bras. Ictiol. 88:7-8. http://www.sbi.bio.br/boletins/BOLETIM88.pdf (último acesso em 23/09/2012).

SMITH, W.S. 1999. Pesque-pague: Uma ameaça à ictiofauna nativa? PUC-SP Ciências Biológicas Ambientais, São Paulo, v.1, n.3, p.313-319.

SMITH, W.S. 2003. Os peixes do rio Sorocaba: a história de uma bacia hidrográfica. Editora TCM, Sorocaba, 163p.

SMITH, W.S. \& MARCIANO, F.T. 2000. A ictiofauna da Floresta Nacional de Ipanema - Iperó, São Paulo, como base para ações de manejo, conservação e educação ambiental. In II Congresso Brasileiro de Unidades de Conservação. Campo Grande, p.409-417.

SMITH, W.S. \& REGALADO, L.B. 2008. Floresta Nacional de Ipanema. Ciênc. Hoje 43(255):70-73.

SMITH, W.S., PETRERE JUNIOR, M. \& BARRELLA, W. 2007. Fish, Sorocaba river sub-basin, state of São Paulo, Brazil. Check List 3(3):282286.

SMITH, W.S., PETRERE JUNIOR, M. \& BARRELLA, W. 2009. The fish community of the Sorocaba River Basin in different habitats (State of São Paulo, Brazil). Braz. J. Biol. 69(4):1015-1025. http://dx.doi.org/10.1590/ S1519-69842009000500005 